\title{
Low-density lipoprotein aggregation predicts adverse cardiovascular events in peripheral artery disease
}

\section{Heffron, Sean P.}

2021-01

Heffron , S P , Ruuth , M K, Xia , Y , Hernandez , G , Äikas , L , Rodriguez , C , Öörni , K \& Berger , J S 2021 , ' Low-density lipoprotein aggregation predicts adverse cardiovascular events in peripheral artery disease ' , Atherosclerosis , vol. 316 , pp. 53-57 . https://doi.org/10.1016/j.atherosclerosis

http://hdl.handle.net/10138/340117

https://doi.org/10.1016/j.atherosclerosis.2020.11.016

cc_by_nc_nd

acceptedVersion

Downloaded from Helda, University of Helsinki institutional repository.

This is an electronic reprint of the original article.

This reprint may differ from the original in pagination and typographic detail.

Please cite the original version. 
8

9

\section{0}

\section{Low-density Lipoprotein Aggregation Predicts Adverse Cardiovascular Events in Peripheral Artery Disease}

Sean P. Heffron MD, MS, MSc, ${ }^{1,2}$ Maija K. Ruuth PhD, ${ }^{4,5}$ Yuhe Xia MS, ${ }^{1}$ Gustavo Hernandez MD, ${ }^{1}$ Lauri Äikäs MSc, ${ }^{4,5}$ Crystalann Rodriguez BS, ${ }^{1}$ Katariina Öörni PhD,,${ }^{4,6 *}$ Jeffrey S. Berger $\mathrm{MD}, \mathrm{MS}^{1,2,3 *}$

NYU Grossman School of Medicine

${ }^{1}$ Leon H. Charney Division of Cardiology

${ }^{2}$ NYU Center for the Prevention of Cardiovascular Disease

${ }^{3}$ Department of Surgery

New York, NY

${ }^{4}$ Wihuri Research Institute

${ }^{5}$ Research Programs Unit, Faculty of Medicine, University of Helsinki

${ }^{6}$ Molecular and Integrative Biosciences Research Programme, Faculty of Biological and Environmental Sciences, University of Helsinki

Helsinki, Finland

${ }^{*}$ Corresponding authors

Address for correspondence:

Jeffrey S. Berger

530 First Ave, Skirball 9R

New York, NY 10016

Tel. 212-263-4004, Fax. 212-263-3988, jeffrey.berger@nyumc.org

Words: 1762

Tables: 3

Figures: 2 


\section{Key words}

34 peripheral artery disease

35 low-density lipoprotein

36 major adverse cardiovascular events

37 risk

38

\section{Abbreviations}

40 atherosclerotic cardiovascular disease (ASCVD)

41 lower extremity revascularization (LER)

42 major adverse cardiovascular events (MACE)

43 myocardial infarction (MI)

$44 \quad$ peripheral artery disease (PAD)

45

46

47

48

49

50

51

52

53

54

55

56 


\section{Abstract}

\section{Aims}

Peripheral artery disease (PAD) is a systemic manifestation of atherosclerosis that is associated with a high risk of major adverse cardiovascular events (MACE). LDL aggregation contributes to atherosclerotic plaque progression and may contribute to plaque instability. We aimed to determine if LDL aggregation is associated with MACE in patients with PAD undergoing lower extremity revascularization (LER).

\section{Methods and Results}

Two hundred thirty-nine patients with PAD undergoing LER had blood collected at baseline and were followed prospectively for MACE (myocardial infarction, stroke, cardiovascular death) for one-year. Mean age was $72.3 \pm 10.9$ years, $32.6 \%$ were female, and LDL-cholesterol was $68 \pm 25 \mathrm{mg} / \mathrm{dL}$. Subject LDL was exposed to sphingomyelinase and LDL aggregate size measured via dynamic light scattering. LDL aggregation was inversely associated with triglycerides, but not associated with demographics, LDL-cholesterol or other risk factors. Maximal LDL aggregation occurred significantly earlier in subjects with PAD than in control subjects without cardiovascular disease (CVD).

$15.9 \%$ of subjects experienced MACE over one year. The $1^{\text {st }}$ tertile (shortest time to maximal aggregation) exhibited significantly higher MACE (25\% vs. $12.5 \%$ in tertile 2 and $10.1 \%$ in tertile $3, p=0.012$ ). After multivariable adjustment for demographics and CVD risk factors, the hazard ratio for MACE in the $1^{\text {st }}$ tertile was 3.05 (95\% Cl $\left.1.30-7.17 ; p=0.01\right)$ compared to the other tertiles. Inclusion of LDL aggregation in the Framingham Heart Study risk calculator for recurrent coronary heart disease events improved the c-index from 0.57 to $0.63(p=0.01)$.

\section{Conclusions}

We show that in the setting of very well controlled LDL-choleseterol, patients with PAD with the most rapid LDL aggregation had a significantly elevated MACE risk following LER even after multivariable adjustment. This measure further improved the classification specificity of an established risk prediction tool. Our findings support broader investigation of this assay for risk stratification in patients with atherosclerotic CVD. 


\section{Introduction}

88 Low-density lipoprotein (LDL) is both a key risk factor and causative factor in atherosclerosis -

89 contributing to lipid-laden plaques with its retention in the arterial intima. ${ }^{1}$ Despite robust

90 lowering of LDL-cholesterol, cardiovascular events remain frequent in patients with

91 atherosclerotic cardiovascular disease (ASCVD). ${ }^{2,3}$ Peripheral artery disease (PAD) is a

92 systemic manifestation of atherosclerosis and those with PAD are at particularly high risk of

93 major adverse cardiovascular events (MACE). ${ }^{4}$ Improved risk stratification and the identification

94 of additional modifiable risk factors for adverse events are needed to improve outcomes in this

95 population. ${ }^{5}$

96 We previously developed an ex vivo assay of LDL aggregation - a process that occurs within

97 and contributes to atherosclerotic plaques. ${ }^{6,7}$ Our work has shown that LDL aggregation differs

98 among individuals independent of plasma LDL-cholesterol concentration and LDL particle size

99 and is predictive of death in coronary artery disease (CAD). ${ }^{8}$ The aims of the present study were

100 to determine if LDL aggregation is associated with MACE in patients with PAD undergoing lower

101 extremity revascularization (LER) and may help identify those at increased risk for adverse

102 outcomes.

103

104

105

106

107

108

109

110

111

112

113

114 


\section{Methods}

116 Clinical study design

117 Men and women scheduled for LER at NYU Tisch Hospital or Bellevue Hospital Center (New

118 York, NY) provided informed consent and were enrolled into the Platelet Activity in Vascular

119 Surgery and Cardiovascular Events study (NCT02106429) under an IRB-approved protocol

120 conforming to the Declaration of Helsinki. Fasting blood samples were collected prior to LER,

121 plasma and serum isolated and immediately frozen at $-80^{\circ} \mathrm{C}$ until analysis. As part of a

122 separate, ongoing protocol, we recruit individuals without known ASCVD from our hospitals to

123 undergo clinical evaluation and blood sampling. Prior to LDL-aggregation analysis, we identified

12419 individuals within this cohort who were of similar sex, race/ethnicity and had similar LDL-C

125 levels as the PAD patients. Samples from these control subjects were processed and analyzed

126 along with samples from PAD subjects in a blinded fashion.

127 BMI was calculated based on pre-operative measures of height and weight. Measurements of

128 circulating lipoproteins were made from serum samples using NMR spectroscopy (LabCorp Inc.,

129 Morrisville, NC) ${ }^{9}$

130 Clinical follow-up of subjects with PAD occurred prospectively at 30-days, 6 months and 12 131 months from the time of operation. Three blinded reviewers (2 cardiologists and 1 vascular 132 surgeon) adjudicated the composite endpoint of all cause death, myocardial infarction (MI), and 133 stroke (both ischemic and hemorrhagic).

134 LDL aggregation assay

135 LDL aggregation was assessed blinded and as previously described. ${ }^{10}$ First, LDL was isolated 136 by sequential ultracentrifugation in $\mathrm{D}_{2} \mathrm{O}$-based buffers as described, ${ }^{11}$ with the exception that 137 samples were first centrifuged twice at a density of $1.019 \mathrm{~g} / \mathrm{ml}$ for efficient removal of all 138 chylomicrons, VLDL, and IDL from the samples. LDL was isolated at a density of $1.063 \mathrm{~g} / \mathrm{ml}$ 139 and, exposed to human recombinant sphingomyelinase produced in-house ${ }^{10,12}$ and LDL 140 aggregate size was measured every 30 minutes for 5 hours via dynamic light scattering (Wyatt 141 DynaPro Plate Reader II; Wyatt Technology, CA). Time-size curves were constructed based on 142 all data points for an individual subject and the inflection point (mid-point of the sigmoidal curve 143 - time point when the aggregation curve has the steepest upward slope) was identified. This 144 inflection point was used for between-subject comparisons. ${ }^{8}$ The intra-assay variability in the 
145 LDL aggregation inflection point was 6.8\% (range 5.9\%-8.2\%) and inter-assay variability was

$1468.1 \%$. The inter-operator variability was $9.6 \%$.

147 Statistical analyses

148 Comparisons between PAD and healthy control subjects were made with two sample t- and

149 Mann-Whitney tests. Multivariable linear regression modeling included age, sex, BMI, statin use, 150 insulin use, clopidogrel use, LDL-C, ApoB, triglycerides, smoking, diabetes, hyperlipidemia and 151 hypertension as covariates. Comparisons between tertiles of subjects with PAD were performed 152 with Kruskal Wallis tests and Jonckheere-Terpstra and Cochrane-Armitage tests for trend.

153 Prediction models were generated using Cox proportional hazards regression. The relation of

154 LDL aggregation inflection point and MACE was presented as Kaplan-Meier curves with

155 inflection point categorized by tertile. The cumulative risk of MACE were calculated in each

156 group and compared using the log-rank test.

157 We tested the capacity of the Framingham Risk Calculator for secondary cardiovascular 158 events ${ }^{13}$ to predict 1-year MACE within this PAD cohort and compared the associated Harrell c159 statistic with and without the addition of LDL aggregation inflection point using the z-score test. 160 A two-tailed $p$-value less than 0.05 was set a priori as the criterion for statistical significance.

161

162 163 164 165 166 167 


\section{Results}

174 Two hundred thirty-nine patients with PAD had LDL aggregation assessed. The mean age was

$17572.3 \pm 10.9$ years and $32.6 \%$ were female. The cohort had a high burden of ASCVD risk factors

176 (Table 1) with more than half having known CAD. LDL aggregation inflection point was not

177 associated with clinical variables, LDL parameters, or other hematologic measures, other than

178 serum triglyceride concentrations (Table 2).

179 In comparison to control subjects recruited from the same hospitals without known ASCVD, 180 patients with PAD exhibited similar LDL-cholesterol and triglyceride levels, but were older, had 181 slightly lower BMI and a greater prevalence of cardiovascular disease risk factors (Table 1). 182 Maximal LDL aggregation occurred significantly earlier in subjects with PAD than in control 183 subjects (Figure 1). This difference persisted after adjustment for demographics, ASCVD risk 184 factors and statin use $(\beta=-0.55(0.25), p=0.03)$.

185 We divided subjects with PAD into tertiles based on LDL aggregation inflection point. There was 186 a linear trend of higher triglycerides across LDL aggregation tertiles (Table 3). However, there 187 were no other differences in demographics, LDL quantity or particle size, medication use or 188 other ASCVD risk factors across LDL aggregation tertiles.

189 Overall, MACE occurred in $15.9 \%$ of PAD subjects at 1 -year. The $1^{\text {st }}$ tertile (shortest time to 190 maximal aggregation rate), exhibited a significantly higher rate of MACE (25\% vs. $12.5 \%$ in 191 tertile 2 and $10.1 \%$ in tertile 3, Figure 2). Data were consistent for each component of the MACE 192 endpoint. After multivariable adjustment for age, sex, race, BMI, smoking status, LDL193 cholesterol, ApoB, triglycerides, diabetes, insulin use, statin use, clopidogrel use and history of 194 prior $\mathrm{MI}$, the hazard ratio for MACE in the $1^{\text {st }}$ tertile was 4.57 (95\% confidence interval $(\mathrm{Cl}) 1.60$ $195-13.01 ; p=0.004)$ compared to the $3^{\text {rd }}$ tertile.

196 The Framingham Heart Study risk equation for recurrent coronary heart disease events had a c197 index of $0.57(95 \% \mathrm{Cl} 0.48,0.67)$ for 1 -year MACE in our cohort of patients with PAD 198 undergoing LER. The addition of LDL aggregation inflection point to this equation increased the 199 c-statistic to $0.63(95 \% \mathrm{Cl} 0.53,0.73 ; p=0.01)$. 
204 The present study, the largest to date employing the LDL aggregation assay, is the first study to 205 assess LDL aggregation in patients with PAD and the first prospective study to evaluate the 206 capacity of this measure in risk stratification. We show that in the setting of very well controlled LDL-cholesterol (mean LDL-cholesterol was $68 \pm 25 \mathrm{mg} / \mathrm{dL}$ ), and after correction for other ASCVD risk factors, patients with the most rapid LDL aggregation had a significantly elevated risk of MACE over one year following LER. This measure further improved the classification specificity within this cohort of an established risk prediction tool. Our findings support broader investigation of this assay for risk stratification in patients with ASCVD.

212

213

214

215

216

217

218

219

220

221

222

223

224

225

226

227

Patients with PAD undergoing LER are at very high risk for MACE, ${ }^{4}$ as evidenced by the $15.9 \%$ event rate in our cohort. Understanding potential mechanisms and identifying those at increased risk may help improve outcomes in this population. We and others previously showed that aggregated LDL is present in human atherosclerotic plaques and that sphingomyelinase catalyzes the aggregative process. ${ }^{6,14}$ We subsequently developed a highly reliable assay of $e x$ vivo LDL aggregation to human recombinant sphingomyelinase ${ }^{8}$ and demonstrated a difference in LDL aggregation between subjects with stable CAD and those who would die from CAD over an average of 2.5 years of follow-up. ${ }^{8}$ Beyond stimulating LDL retention and overall plaque burden, LDL aggregation may also contribute to plaque rupture and adverse outcomes. Aggregated LDL isolated from human atherosclerotic lesions activates the inflammasome ${ }^{6}$ and MMP-7 secretion from macrophages is induced by LDL treated with sphingomyelinase. ${ }^{8}$ Both of these activities are associated with plaque instability and rupture and may mechanistically link elevated LDL aggregation and atherothrombotic events. Accordingly, the tertile of most rapid LDL aggregation in our cohort experienced a 3-fold elevated risk of MACE over one year even after correction for traditional risk factors. Incorporation of LDL aggregation inflection point into an established risk equation improved the c-statistic by more than $10 \%$, demonstrating the potential capacity of this assay to discriminate those at elevated risk.

Given the mechanistic rationale for the association of LDL aggregation with higher rates of atherothrombosis, this measure is potentially a modifiable causative factor, in addition to a marker of risk. While a recent randomized trial did not find that dietary supplementation with alpha-linoleic acid or increased fish consumption affected LDL aggregation, ${ }^{15}$ other studies suggest that adopting a Nordic-style diet, adding plant stanols to the diet, or the use of a PCSK9 inhibitor reduce LDL aggregation in association with altered LDL particle sphingomyelin content. $^{8,16}$ Notably, PCSK9 inhibition has been shown to reduce MACE in individuals with 
$\mathrm{PAD}^{17}$ and we have demonstrated a strong association of dietary quality with PAD. ${ }^{18,19}$ Whether

237 PCSK9 inhibition and/or intensive dietary modification might improve outcomes in patients with

238 PAD with rapid LDL aggregation who are at increased risk for MACE, despite well controlled

239 LDL-cholesterol, will require randomized trials.

240 In agreement with our previous reports, LDL aggregation was not associated with LDL-

241 cholesterol or LDL particle size in the present study. LDL aggregation inflection point did

242 inversely correlate with serum triglycerides, also in accordance with prior studies. ${ }^{8}$ The reasons

243 for this seemingly paradoxical association are unclear. Serum triglyceride level reflects

244 triglyceride content of LDL particles and greater LDL triglyceride content has been shown to

245 associate with reduced aggregation-susceptibility of LDL particles. Increases in LDL-

246 triglycerides are associated with greater proportions of phosphatidylcholines and lower

247 proportions of sphingomyelins in the surface monolayer of LDL particles. We previously showed

248 that changes in the proportions of these two types of phospholipids control the aggregation of

249 LDL particles. ${ }^{8}$ LDL from human $A P O B$ transgenic/LDLr/-/Soat2-/mice exhibiting increased

250 LDL-triglyceride content is extremely resistant to sphingomyelinase-induced LDL aggregation. ${ }^{8}$

251 Whether LDL triglyceride content independently affects LDL aggregative potential is the focus of

252 ongoing studies.

253 Our study has several limitations. Our cohort only contains individuals with severe PAD

254 requiring LER. Whether LDL aggregation provides similar risk prediction in a broader population

255 of patients with PAD will require additional investigation. Additionally, while our subjects came

256 from two separate hospitals, they represented a single locale and were predominantly

257 Caucasian males. We recently reported differences in LDL aggregation between races, with

258 South Asians exhibiting more rapid aggregation than Caucasians. ${ }^{10}$ Further studies of more 259 diverse cohorts, including greater numbers of women - in whom PAD is most prevalent ${ }^{20,21}$

260 are necessary before extrapolating our findings.

261 In conclusion, we show that patients with PAD undergoing LER with more rapid LDL

262 aggregation have a significantly elevated risk of MACE over one year and that this measure

263 improves risk prediction in this group. These findings support broader investigation of this assay

264 for risk stratification and potential intervention in patients with ASCVD. 


\section{Funding}

268 SPH was supported by NIH (HL135398). KÖ was supported by The Academy of Finland

269 (\#315568), the Finnish Foundation for Cardiovascular Research, and Aarne Koskelo

270 Foundation. JSB was supported by NIH (HL139909 and HL144993).

271

272 Acknowledgements

273 We are grateful for the technical assistance of Maija Atuegwu.

274

275 Disclosures

276 KÖ and MKR have applied for a patent on the LDL aggregation assay. The authors have no 277 other disclosures to report. 


\section{References}

279 1. Ference BA, Ginsberg HN, Graham I, Ray KK, Packard CJ, Bruckert E, Hegele RA, 280 Krauss RM, Raal FJ, Schunkert H, Watts GF, Borén J, Fazio S, Horton JD, Masana L, Nicholls SJ, Nordestgaard BG, van de Sluis B, Taskinen MR, Tokgözoglu L, Landmesser U, Laufs U, Wiklund O, Stock JK, Chapman MJ and Catapano AL. Low-density lipoproteins cause atherosclerotic cardiovascular disease. 1. Evidence from genetic, epidemiologic, and clinical studies. A consensus statement from the European Atherosclerosis Society Consensus Panel. Eur Heart J. 2017;38:2459-2472.

2. Catapano AL, Graham I, De Backer G, Wiklund O, Chapman MJ, Drexel H, Hoes AW, Jennings CS, Landmesser U, Pedersen TR, Reiner Ž, Riccardi G, Taskinen MR, Tokgozoglu L, Verschuren WMM, Vlachopoulos C, Wood DA, Zamorano JL and Cooney MT. 2016 ESC/EAS Guidelines for the Management of Dyslipidaemias. Eur Heart J. 2016;37:2999-3058.

3. Sabatine MS, Giugliano RP, Keech AC, Honarpour N, Wiviott SD, Murphy SA, Kuder JF, Wang H, Liu T, Wasserman SM, Sever PS and Pedersen TR. Evolocumab and Clinical Outcomes in Patients with Cardiovascular Disease. The New England journal of medicine. 2017;376:1713-1722.

4. Emdin CA, Anderson SG, Callender T, Conrad N, Salimi-Khorshidi G, Mohseni H, Woodward $\mathrm{M}$ and Rahimi K. Usual blood pressure, peripheral arterial disease, and vascular risk: cohort study of 4.2 million adults. BMJ (Clinical research ed). 2015;351 :h4865-h4865. 5. Shammas NW. Epidemiology, classification, and modifiable risk factors of peripheral arterial disease. Vascular health and risk management. 2007;3:229-34.

6. Lehti S, Nguyen SD, Belevich I, Vihinen H, Heikkilä HM, Soliymani R, Käkelä R, Saksi J, Jauhiainen M, Grabowski GA, Kummu O, Hörkkö S, Baumann M, Lindsberg PJ, Jokitalo E, Kovanen PT and Öörni K. Extracellular Lipids Accumulate in Human Carotid Arteries as Distinct Three-Dimensional Structures and Have Proinflammatory Properties. The American journal of pathology. 2018;188:525-538.

7. Borén J, Chapman MJ, Krauss RM, Packard CJ, Bentzon JF, Binder CJ, Daemen MJ, Demer LL, Hegele RA, Nicholls SJ, Nordestgaard BG, Watts GF, Bruckert E, Fazio S, Ference BA, Graham I, Horton JD, Landmesser U, Laufs U, Masana L, Pasterkamp G, Raal FJ, Ray KK, Schunkert H, Taskinen MR, van de Sluis B, Wiklund O, Tokgozoglu L, Catapano AL and Ginsberg HN. Low-density lipoproteins cause atherosclerotic cardiovascular disease: pathophysiological, genetic, and therapeutic insights: a consensus statement from the European Atherosclerosis Society Consensus Panel. Eur Heart J. 2020.

8. Ruuth M, Nguyen SD, Vihervaara T, Hilvo M, Laajala TD, Kondadi PK, Gisterå A, Lähteenmäki H, Kittilä T, Huusko J, Uusitupa M, Schwab U, Savolainen MJ, Sinisalo J, Lokki ML, Nieminen MS, Jula A, Perola M, Ylä-Herttula S, Rudel L, Öörni A, Baumann M, Baruch A, Laaksonen R, Ketelhuth DFJ, Aittokallio T, Jauhiainen M, Käkelä R, Borén J, Williams KJ, Kovanen PT and Öörni K. Susceptibility of low-density lipoprotein particles to aggregate depends on particle lipidome, is modifiable, and associates with future cardiovascular deaths. European heart journal. 2018;39:2562-2573.

9. Manmadhan A, Lin BX, Zhong J, Parikh M, Berger JS, Fisher EA and Heffron SP. Elevated GlycA in severe obesity is normalized by bariatric surgery. Diabetes, obesity \& metabolism. 2019;21:178-182.

10. Ruuth M, Janssen LGM, Äikäs L, Tigistu-Sahle F, Nahon KJ, Ritvos O, Ruhanen H, Käkelä R, Boon MR, Öörni K and Rensen PCN. LDL aggregation susceptibility is higher in healthy South Asian compared with white Caucasian men. J Clin Lipidol. 2019;13:910-919.e2. 11. Hallberg C, Hådén M, Bergström M, Hanson G, Pettersson K, Westerlund C, Bondjers G, Ostlund-Lindqvist AM and Camejo G. Lipoprotein fractionation in deuterium oxide gradients: a procedure for evaluation of antioxidant binding and susceptibility to oxidation. Journal of lipid research. 1994;35:1-9. 
12. Bordeaux BC, Qayyum R, Yanek LR, Vaidya D, Becker LC, Faraday N and Becker DM. Effect of obesity on platelet reactivity and response to low-dose aspirin. Preventive cardiology. 2010;13:56-62.

13. D'Agostino RB, Russell MW, Huse DM, Ellison RC, Silbershatz H, Wilson PW and Hartz SC. Primary and subsequent coronary risk appraisal: new results from the Framingham study. American heart journal. 2000;139:272-81. aorta and human atherosclerotic lesions hydrolyze the sphingomyelin of retained low-density lipoprotein. Proposed role for arterial-wall sphingomyelinase in subendothelial retention and aggregation of atherogenic lipoproteins. J Clin Invest. 1996;98:1455-64.

15. Manninen S, Lankinen M, Erkkilä A, Nguyen SD, Ruuth M, de Mello V, Öörni K and Schwab U. The effect of intakes of fish and Camelina sativa oil on atherogenic and antiatherogenic functions of LDL and HDL particles: A randomized controlled trial. Atherosclerosis. 2019;281:56-61.

16. Ruuth M AL, Tigistu-Sahle F, Kakela R, Lindholm H, Simonen P, Kovanen PT, Gylling $H$, Oorni K. Plant stanol esters reduce LDL aggregation by altering LDL surface lipids. The BLOOD FLOW randomized intervention study. Arteriosclerosis Thrombosis and Vascular Biology. 2020. 17. Bonaca MP, Nault P, Giugliano RP, Keech AC, Pineda AL, Kanevsky E, Kuder J, Murphy SA, Jukema JW, Lewis BS, Tokgozoglu L, Somaratne R, Sever PS, Pedersen TR and Sabatine MS. Low-Density Lipoprotein Cholesterol Lowering With Evolocumab and Outcomes in Patients With Peripheral Artery Disease: Insights From the FOURIER Trial (Further Cardiovascular Outcomes Research With PCSK9 Inhibition in Subjects With Elevated Risk). Circulation. 2018;137:338-350.

18. Heffron SP, Rockman CB, Gianos E, Guo Y and Berger JS. Greater frequency of nut consumption is associated with lower prevalence of peripheral arterial disease. Prev Med. 2015;72:15-8.

19. Heffron SP, Rockman CB, Adelman MA, Gianos E, Guo Y, Xu JF and Berger JS. Greater Frequency of Fruit and Vegetable Consumption Is Associated With Lower Prevalence of Peripheral Artery Disease. Arterioscler Thromb Vasc Biol. 2017;37:1234-1240. 20. Sigvant B, Wiberg-Hedman K, Bergqvist D, Rolandsson O, Andersson B, Persson E and Wahlberg E. A population-based study of peripheral arterial disease prevalence with special focus on critical limb ischemia and sex differences. J Vasc Surg. 2007;45:1185-91. 21. Zheng ZJ, Rosamond WD, Chambless LE, Nieto FJ, Barnes RW, Hutchinson RG, Tyroler HA and Heiss G. Lower extremity arterial disease assessed by ankle-brachial index in a middle-aged population of African Americans and whites: the Atherosclerosis Risk in Communities (ARIC) Study. Am J Prev Med. 2005;29:42-9. 


\section{Figure Legends}

372 Figure 1. (A) Representative aggregation curves of a PAD subject (black squares) and healthy 373 control subject (gray circles) with inflection point highlighted. (B) LDL aggregation inflection point 374 in all subjects with peripheral artery disease $(n=239)$ and control subjects without known 375 atherosclerotic cardiovascular disease $(n=19)$. Comparison with two sample t-tests.

376

377

378 379

380

381

382

383

384

385

386

387

388

389

390

391

392

393

394

395

396

397

398

399

400

401

99

Figure 2. Kaplan-Meier curves for major adverse cardiovascular events over one year following lower extremity revascularization in 239 subjects stratified by tertiles of LDL aggregation inflection point. Cumulative risk of MACE between tertiles was compared using the log-rank test.

79

0

81

2

4

85

87

8

89

91

92

94

95

96

97

98

.


Table 1. Descriptive characteristics of subjects with peripheral artery disease and control subjects without known atherosclerotic cardiovascular disease.

\begin{tabular}{|c|c|c|c|}
\hline & No ASCVD & PAD & P-value \\
\hline & $n=19$ & $\mathrm{n}=239$ & \\
\hline Age, mean (SD), years & $63.2(6.3)$ & $72.3(10.9)$ & $<0.001$ \\
\hline Female sex, n (\%) & $7(37 \%)$ & $78(32.6 \%)$ & 0.81 \\
\hline BMI, mean (SD), kg/m² & $29.9(5.1)$ & $26.7(5.3)$ & 0.01 \\
\hline LDL-cholesterol, mean (SD), mg/dL & $77(42)$ & $68(25)$ & 0.47 \\
\hline HDL-cholesterol, mean (SD), mg/dL & $55(17)$ & $43(13)$ & 0.006 \\
\hline Triglycerides, median [range], mg/dL & $116[35,259]$ & $94[31,349]$ & 0.20 \\
\hline Hypertension, n (\%) & $11(57.9)$ & $205(85.8)$ & 0.002 \\
\hline Hyperlipidemia, n (\%) & $12(63.2)$ & $177(74.1)$ & 0.34 \\
\hline Diabetes, n (\%) & $3(15.8)$ & $118(49.4)$ & 0.002 \\
\hline Insulin use, n (\%) & $0(0.0)$ & $75(31.4)$ & - \\
\hline Coronary artery disease, n (\%) & $0(0.0)$ & $133(55.6)$ & - \\
\hline Prior myocardial infarction, $\mathrm{n}(\%)$ & $0(0.0)$ & $55(23.0)$ & - \\
\hline Current smokers, n (\%) & $1(5.3)$ & $38(15.9)$ & 0.20 \\
\hline Statin use, n (\%) & $11(57.9)$ & $193(80.7)$ & 0.02 \\
\hline Aspirin use, $\mathrm{n}(\%)$ & $10(52.6)$ & $208(87.0)$ & $<0.001$ \\
\hline Clopidogrel use, n (\%) & $0(0.0)$ & $90(37.7)$ & - \\
\hline
\end{tabular}

404 SI conversion factors: To convert cholesterol to $\mathrm{mmol} / \mathrm{L}$, multiply values by 0.0259 .

ASCVD - atherosclerotic cardiovascular disease

$\mathrm{BMI}$ - body mass index

LDL - low density lipoprotein

$\mathrm{HDL}$ - high density lipoprotein

PAD - peripheral artery disease

SD - standard deviation 
411 Table 2. Correlations of clinical and hematologic variables with LDL aggregation inflection point.

\begin{tabular}{|l|c|c|}
\hline & $\mathbf{r}=$ & P-value \\
\hline Age & -0.03 & 0.69 \\
\hline BMI & -0.08 & 0.21 \\
\hline White blood cells & 0.02 & 0.83 \\
\hline Neutrophils & -0.002 & 0.98 \\
\hline Monocytes & -0.08 & 0.22 \\
\hline Hemoglobin A1c & -0.03 & 0.73 \\
\hline Total cholesterol & 0.004 & 0.96 \\
\hline LDL-cholesterol & -0.05 & 0.44 \\
\hline LDL-particles & 0.03 & 0.65 \\
\hline LDL-particle size & -0.08 & 0.22 \\
\hline Triglycerides & 0.14 & 0.03 \\
\hline HDL-cholesterol & -0.02 & 0.79 \\
\hline Creatinine & 0.08 & 0.25 \\
\hline Systolic blood pressure & -0.03 & 0.35 \\
\hline
\end{tabular}

$412 \mathrm{BMI}$ - body mass index

413 LDL - low density lipoprotein

$414 \quad \mathrm{HDL}-$ high density lipoprotein

415

416

417

418

419

420

421

422

423

424

425

426

427

428

429 
Table 3. Descriptive characteristics of tertiles of LDL aggregation in subjects with peripheral artery disease undergoing lower extremity revascularization

\begin{tabular}{|c|c|c|c|c|}
\hline & $\begin{array}{l}\text { LDL- } \\
\text { aggregation } \\
\text { Tertile } 1\end{array}$ & $\begin{array}{l}\text { LDL- } \\
\text { aggregation } \\
\text { Tertile } 2\end{array}$ & $\begin{array}{c}\text { LDL- } \\
\text { aggregation } \\
\text { Tertile } 3\end{array}$ & $\begin{array}{l}\text { P-value } \\
\text { for trend }\end{array}$ \\
\hline & $\mathrm{n}=80$ & $\mathrm{n}=79$ & $\mathrm{n}=80$ & \\
\hline Age, mean (SD), years & $73.5(12.2)$ & $72.7(9.8)$ & $70.3(11.0)$ & 0.15 \\
\hline Female sex, $\mathrm{n}(\%)$ & $28(35.0)$ & $28(35.4)$ & $22(27.5)$ & 0.31 \\
\hline BMI, mean (SD), $\mathrm{kg} / \mathrm{m}^{2}$ & $26.8(5.6)$ & $27.2(5.1)$ & $26.3(5.4)$ & 0.58 \\
\hline LDL-cholesterol, mean (SD), mg/dL & $71(27)$ & $75(24)$ & $68(23)$ & 0.97 \\
\hline ApoB, mean (SD), mg/dL & $109(27)$ & $107(24)$ & $107(22)$ & 0.93 \\
\hline LDL-particles, mean (SD), nmol/L & $1147(379)$ & $1232(358)$ & $1145(323)$ & 0.56 \\
\hline LDL-particle size, mean (SD), nm & $20.5(0.6)$ & $20.5(0.6)$ & $20.3(0.6)$ & 0.10 \\
\hline HDL-cholesterol, mean (SD), mg/dL & $41(11)$ & $43(12)$ & $41(10)$ & 0.23 \\
\hline Triglycerides, median [range], mg/dL & $83[31,290]$ & $97[35,236]$ & $118[41,349]$ & $<0.01$ \\
\hline Hypertension, n (\%) & $68(85.0)$ & $69(87.3)$ & $68(85.0)$ & 0.85 \\
\hline Hyperlipidemia, n (\%) & $63(78.8)$ & $55(69.6)$ & $59(73.8)$ & 0.42 \\
\hline Diabetes, n (\%) & $36(45.0)$ & $36(45.6)$ & $46(57.5)$ & 0.08 \\
\hline Insulin use, n (\%) & $28(35.0)$ & $26(32.9)$ & $21(26.3)$ & 0.25 \\
\hline Coronary artery disease, n (\%) & $48(60.0)$ & $43(54.4)$ & $42(52.5)$ & 0.43 \\
\hline Prior myocardial infarction, $\mathbf{n}(\%)$ & $16(20.0)$ & $20(25.3)$ & $19(23.8)$ & 0.57 \\
\hline Current smokers, n (\%) & $9(11.3)$ & $12(15.2)$ & $17(21.3)$ & 0.09 \\
\hline Statin use, $\mathrm{n}(\%)$ & $65(81.5)$ & $62(78.5)$ & $66(82.5)$ & 0.65 \\
\hline Aspirin use, $\mathrm{n}(\%)$ & $63(78.8)$ & $66(83.5)$ & $69(86.3)$ & 0.15 \\
\hline Clopidogrel use, n (\%) & $30(37.5)$ & $26(32.9)$ & $34(42.5)$ & 0.58 \\
\hline
\end{tabular}

SI conversion factors: To convert cholesterol to $\mathrm{mmol} / \mathrm{L}$, multiply values by 0.0259 .

ASCVD - atherosclerotic cardiovascular disease

$\mathrm{BMI}$ - body mass index

LDL - low density lipoprotein

HDL - high density lipoprotein

SD - standard deviation 
(A)

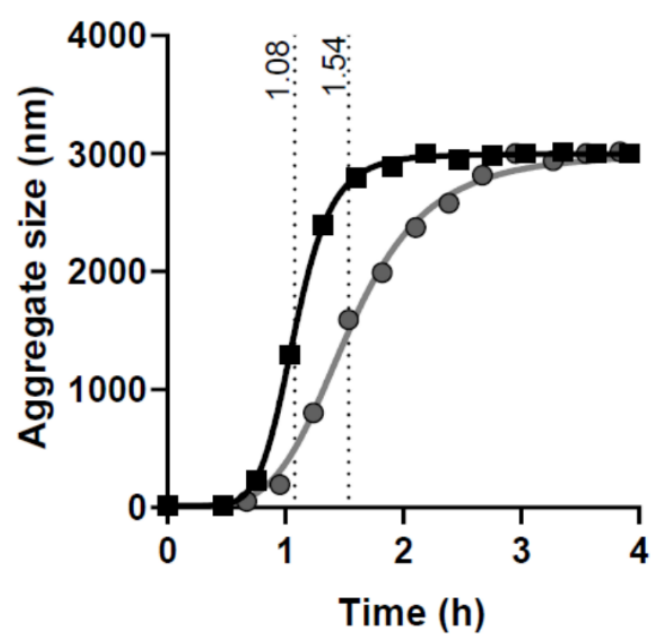

(B)

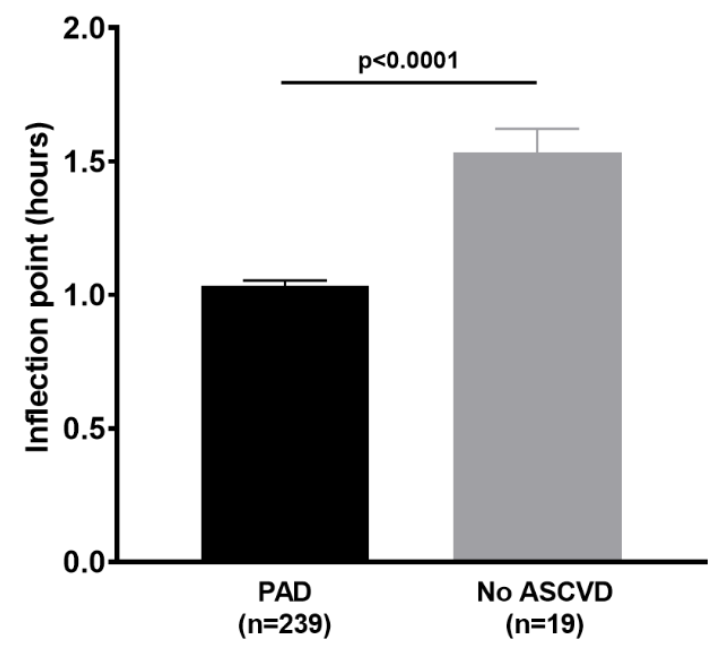

442

443 Figure 1. (A) Representative aggregation curves of a PAD subject (black squares) and healthy 444 control subject (gray circles) with inflection point highlighted. (B) LDL aggregation inflection point 445 in all subjects with peripheral artery disease $(n=239)$ and control subjects without known 446 atherosclerotic cardiovascular disease $(n=19)$. Comparison with two sample t-tests. 


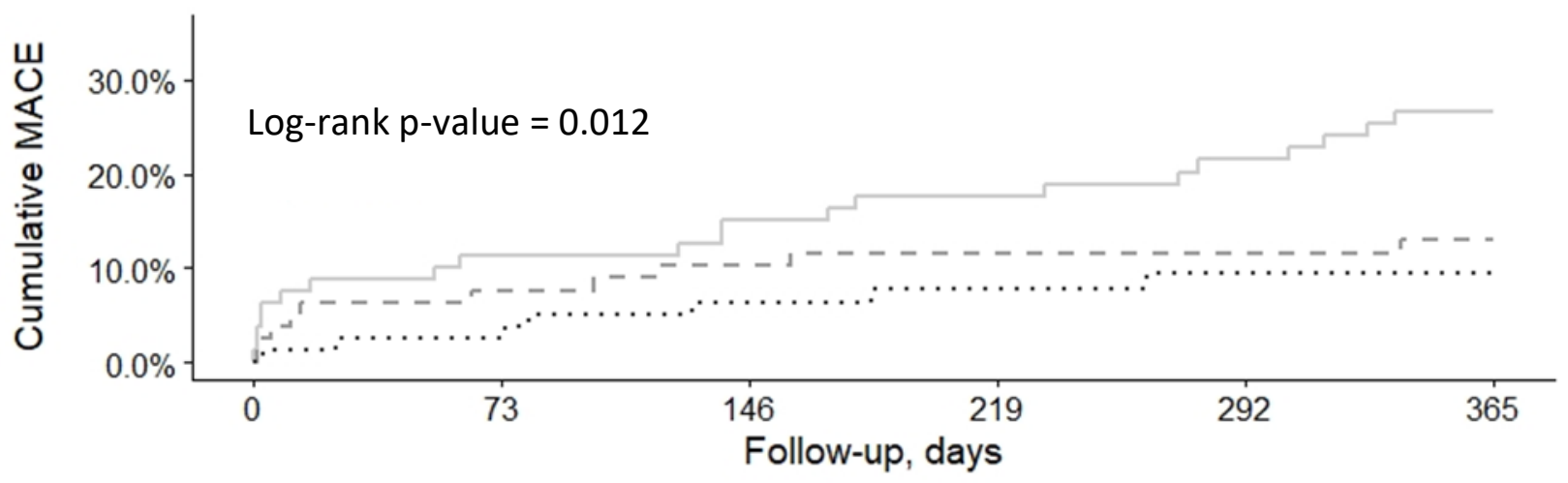

\begin{tabular}{|c|c|c|c|c|c|c|}
\hline \multirow{5}{*}{ 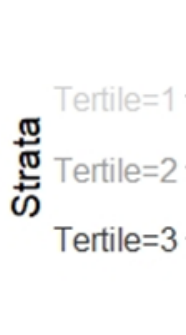 } & \multicolumn{6}{|c|}{ Number at risk } \\
\hline & 80 & 70 & 67 & 65 & 61 & 55 \\
\hline & 79 & 72 & 70 & 66 & 59 & 53 \\
\hline & 80 & 78 & 72 & 62 & 54 & 47 \\
\hline & 0 & 73 & 146 & 219 & 292 & 365 \\
\hline
\end{tabular}

450

451

452

453

Figure 2. Kaplan-Meier curves for major adverse cardiovascular events over one year following lower extremity revascularization in 239 subjects stratified by tertiles of LDL aggregation inflection point. Cumulative risk of MACE between tertiles was compared using the log-rank test. 